Supporting Information

\title{
Graphene-Insulator-Semiconductor Ultraviolet Light-Responsive Nitride LEDs for Multi-Applications
}

Meng-Jer Wu*, Yuejin Wang $\$$, , Shang-Cheng Wu, Yu-Chuan Chien, Chi-Yuan Chang, Jyun-Han Chen", Yu-Ming Liao, Tai-Yuan Lin\& ${ }^{\star}$, Shuping Li ${ }^{\S}$, Duanjun Cai ${ }^{\S}{ }^{*}$, Junyong Kang§, and Yang-Fang Chen*

*E-mail: yfchen@phys.ntu.edu.tw; dcai@xmu.edu.cn

Department of Physics, National Taiwan University, Taipei 10617, Taiwan

KEYWORDS: Ultraviolet, Graphene-Insulator-Semiconductor, Light-EmittingDiode, Photodetector, Double-Side Emission 


\section{Contents:}

A: Mechanism of surface plasma enhancement effect

B: Electroluminescence intensity of the ultraviolet LED with different thickness

of metal thin films

C: SEM image of monolayer graphene on the device

D: AIGaN composition analysis

E: Quality analysis and $\mathrm{SEM}$ images of top side of $\mathrm{SiO}_{2}$ and $\mathrm{AIGaN}$ on the device

F: Dependence of the light intensity on the $\mathrm{SiO}_{2}$ barrier thickness 


\section{A: Mechanism of surface plasma enhancement effect}

Surface plasmons signifies the collective electronic oscillations at the interface between metals and dielectric materials. When the frequency of the incident light is in agreement with the resonance frequency of the surface plasmons, localized electromagnetic fields near the metal are enhanced drastically. In our work, aluminum was chosen for surface plasmon enhancement, because the surface plasmon resonance wavelength of $\mathrm{Al}$ is located in the UV region, which matches well with our devices as shown in Figure 5(c). The induced near fields around the Al layer could strongly interact with the active layer. Therefore, to realize surface plasmon enhanced GIS LEDs, the thickness of insulating layer and graphene is critical for our GIS LEDs. The penetration depth of the surface plasmon near field into the $\mathrm{AlGaN}$ is given by the following expression:

$$
\mathrm{Z}=\lambda / 2 \pi\left[\frac{\left(\epsilon_{A l G a N}^{\prime}-\epsilon_{A l}^{\prime}\right)}{\epsilon_{A l G a N}^{\prime 2}}\right]^{1 / 2}
$$

where $\epsilon_{A l G a N}^{\prime}$ and $\epsilon_{A l}^{\prime}$ are the real parts of the dielectric constants of the AlGaN and $\mathrm{Al}$, respectively. $\mathrm{AlGaN}$ is set with dielectric constant of 7 . The penetration depth was estimated to be $24.3 \mathrm{~nm}$ at the wavelength of $328 \mathrm{~nm}$ for our GIS LEDs. Besides, the presence of $\mathrm{SiO}_{2}$ successfully prevents transport of the electrons and holes from AlGaN to the aluminum layer. Therefore, electron-hole pairs located within the near-field of the $\mathrm{AlGaN}$ surface will recombine radiatively to amplify the intensity of output light, and the hot-carrier injection is unlikely. 


\section{B: Electroluminescence intensity of the ultraviolet LED with different thickness}

\section{of metal thin films}

The table shows the electroluminescence intensity of the ultraviolet LED with different thickness of metal thin films; the device with $3 \mathrm{~nm} \mathrm{Al}$ thin film has the most strong electroluminescence intensity.

\begin{tabular}{|c|c|c|c|c|c|}
\hline $\begin{array}{c}\text { Thickness } \\
\text { Metal }\end{array}$ & $1 \mathrm{~nm}$ & $2 \mathrm{~nm}$ & $3 \mathrm{~nm}$ & $4 \mathrm{~nm}$ & $5 \mathrm{~nm}$ \\
\hline $\begin{array}{c}\mathrm{Au} \\
\text { nanoparticles }\end{array}$ & 304 & 303 & 283 & 276 & 243 \\
\hline $\begin{array}{c}\mathrm{Ag} \\
\text { nanoparticles }\end{array}$ & 322 & 234 & 130 & 140 & 135 \\
\hline $\begin{array}{c}\mathrm{Al} \\
\text { nanoparticles }\end{array}$ & 348 & 481 & 562 & 530 & $\begin{array}{c}374 \\
\text { (arb. unit) }\end{array}$ \\
\hline
\end{tabular}

Table S1. Table shows the electroluminescence intensity of the ultraviolet LED with different thickness of metal thin films. 


\section{C: SEM image of monolayer graphene on device}

SEM image of monolayer graphene on the device is shown in Figure S1. The continuous and crack-free surface shown in the figure confirms the uniform deposition of monolayer graphene.

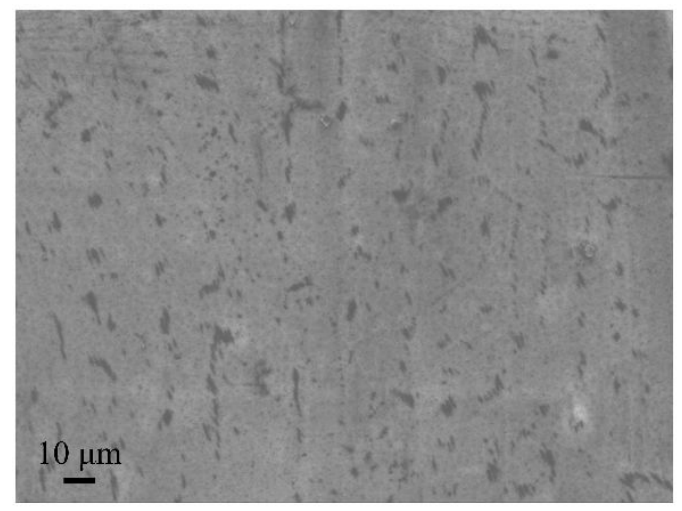

Figure S1. SEM image of monolayer graphene on device 
D: AIGaN composition analysis

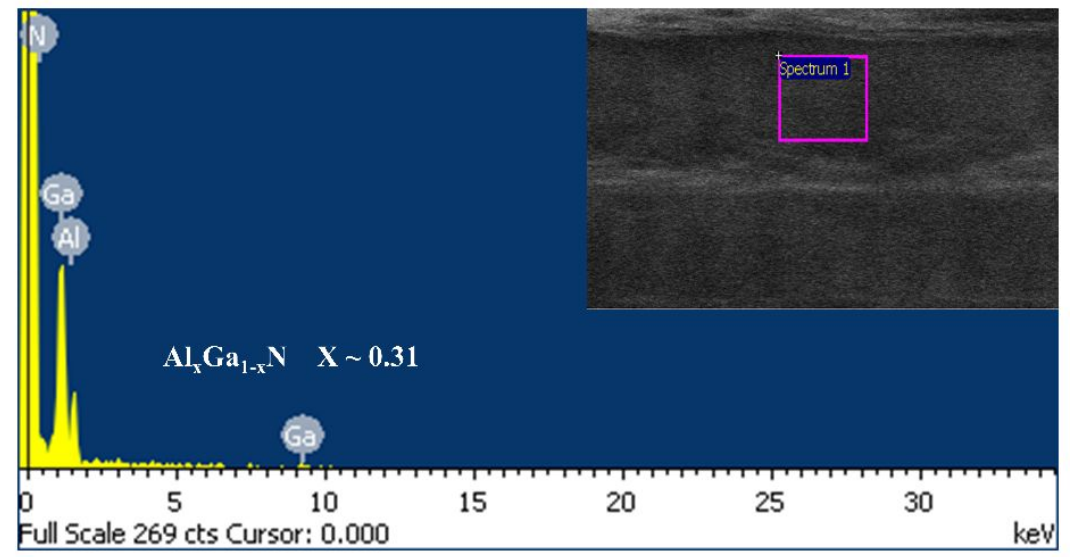

Figure S2. EDS spectrum for cross section of the AlGaN active layer. 
E: Quality analysis and SEM images of top side of $\mathrm{SiO}_{2}$ and $\mathrm{AlGaN}$ on the device The SEM image of $\mathrm{SiO}_{2}$ layer on the device is shown in Figure $\mathrm{S} 3$. The continuous and crack-free surface shown in the figure confirms the uniform deposition of $\mathrm{SiO}_{2}$ layer, which enables to efficiently reduce the leakage current and the carriers can accumulate at the $\mathrm{n}-\mathrm{AlGaN} / \mathrm{AlN}$ interface. In addition, high-quality semiconductor film is the key to fabricate high performance devices. Figure S4 shows the SEM image of the top surface of $\mathrm{AlGaN}$ thin film, which only exhibits polycrystalline dislocation boundaries. The structure distortions and surface cracks caused by the lattice mismatching were not found. In other words, the underlying $\mathrm{AlGaN}$ buffer layers effectively release the stress at the interface and reduce the defects during deposition process. 


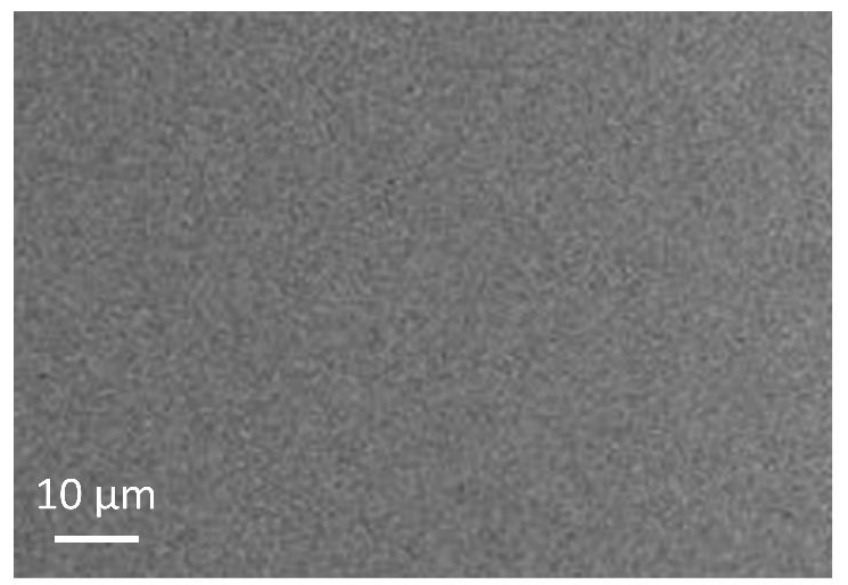

Figure S3. SEM image of $\mathrm{SiO}_{2}$ layer on the device.

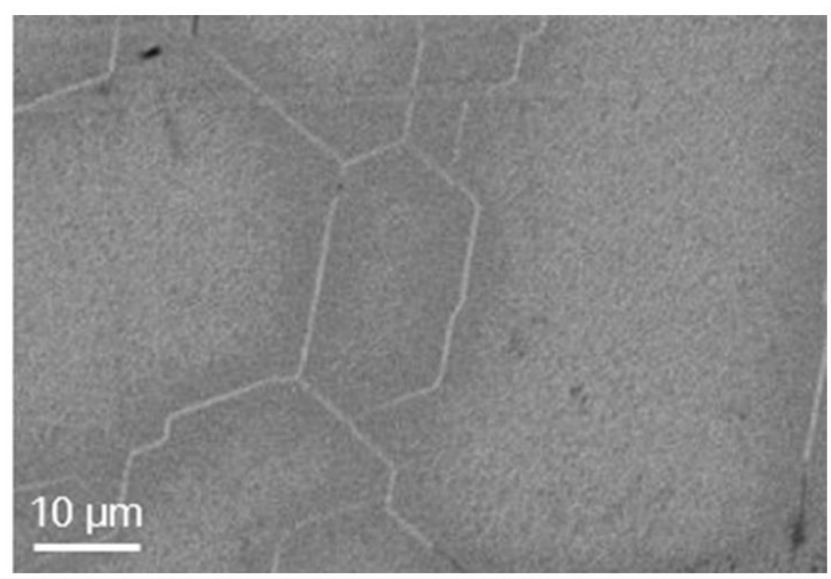

Figure S4. SEM image of AlGaN layer on the device. 
F: Dependence of the light intensity on the $\mathrm{SiO}_{2}$ barrier thickness

Light intensity for different barrier thickness has been presented in Figure S5. Our GIS ultraviolet LED with $8 \mathrm{~nm} \mathrm{SiO}_{2}$ barrier shows the best electroluminescence performance compared to device with thickness less than $6 \mathrm{~nm}$ and larger than $10 \mathrm{~nm}$. When the barrier thickness is too large, it will reduce the probability of quantum tunneling and decrease emitting light intensity. The main reason for the relatively low performance for the device with $\mathrm{SiO}_{2}$ thickness $\sim 6 \mathrm{~nm}$ or less is that when the thickness of the insulating layer is too thin, which can't form a uniform thin film and large leakage current is obtained. In other words, the carriers are hard to accumulate at the n$\mathrm{AlGaN} / \mathrm{AlN}$ interface, which would reduce the radiative recombination drastically.

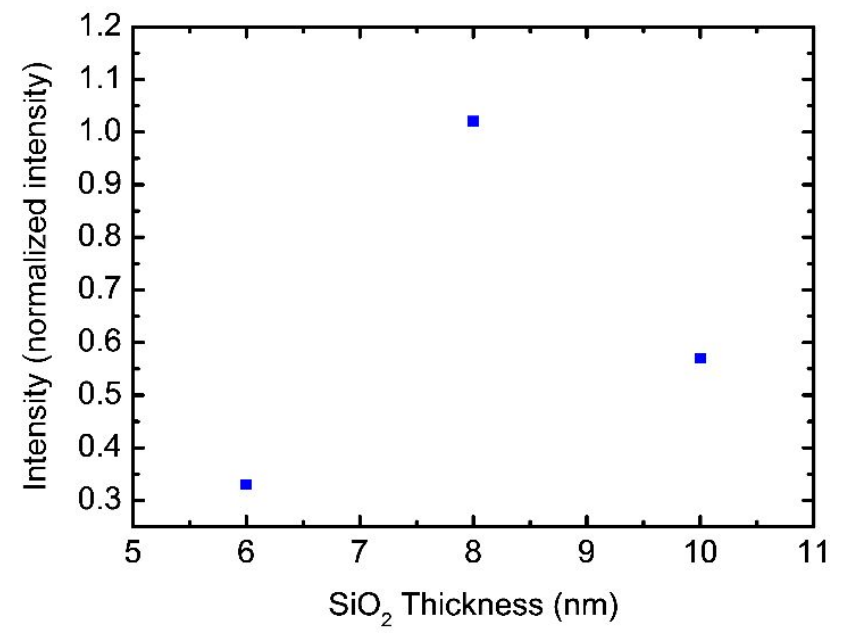

Figure S5. Normalized electroluminescence intensity with different $\mathrm{SiO}_{2}$ thickness. 